\title{
On the Placement of an Obstacle or a Well so as to Optimize the Fundamental Eigenvalue
}

\author{
Evans M. Harrell II* \\ Pawel Kröger** \\ Kazuhiro Kurata ${ }^{* * *}$
}

\begin{abstract}
We investigate how to place an obstacle $B$ within a domain $\Omega$ in Euclidean space so as to maximize or minimize the principal Dirichlet eigenvalue for the Laplacian on $\Omega \backslash B$. The shape of $B$ is fixed a priori (usually as a ball), and only its position varies. We establish that for a certain class of domains the minimizing $B$ is in contact with $\partial \Omega$, while the maximizing $B$ is in the interior, typically at the center (supposing that the domain is sufficiently symmetric for this statement to be meaningful). Under special circumstances we can characterize the optimizing configurations with multiple obstacles. Our method relies on the Hadamard perturbation formula and a moving plane analysis.

Similar facts are proved when the hard obstacle is replaced by a central nonnegative potential function supported in $B$, and we consider the Schrödinger operator with this potential. Complementary facts are proved when the obstacle is replaced by a central nonpositive potential function.
\end{abstract}

\section{Introduction}

In this article we study how to minimize or maximize the fundamental eigenvalue of the Laplacian or Schrödinger operator defined within a fixed, bounded, open domain $\Omega$, with zero Dirichlet boundary conditions on the boundary. Inside this domain we shall place an obstacle or a well, the position of which is under our control, and our goal is to locate the optimal position of the piece under our control.

The obstacles we consider may be hard, by which we mean that zero Dirichlet conditions are additionally imposed on the boundary of some open subset $B$ of $\Omega$, or they may be soft, by which we mean that the operator we analyze is of the fol lowing form:

*harrell@math.gatech.edu, School of Mathematics, Georgia Institute of Technology, Atlanta, GA 30332-0160, USA \& Laboratories MIP and IRSAMC, Université Paul Sabatier, 31062 Toulouse, France. This work was supported by Centre National pour la Recherche Scientifique and by NSF grant DMS-9622730.

**pkroeger@mat.utfsm.cl, Departamento de Matemática, UTFSM, Valparaiso, Chile.

***kurata@comp.metro-u.ac.jp, Department of Mathematics, Tokyo Metropolitan University, Minami-Ohsawa 1-1, Hachioji-shi, Tokyo 192-0397, JAPAN. This work was supported by Grant-inAid for Scientific Research (C) 09640208.

(C) 2000, SIAM. Noncommercial reprinting and electronic distribution permitted under US Copyright Fair Use Provisions. 


$$
-\nabla^{2}+\alpha \chi_{B}(\mathbf{x})
$$

where $\alpha>0$ and $\chi_{B}$ is the indicator function of the region $B$. Lo osely, a hard obstacle corresponds to $\alpha=+\infty$. The term "well" refers to the situation where the constant $\alpha$ in operato $r$ (1.1) is negative. These operators are defined in standard ways [e.g., Da95], and by our sign convention the fundamental eigenvalue with an obstacle is positive and deno ted $\lambda$; in the case of a well, $\lambda$ might be negative. We recall that $\lambda$ is nondegenerate and has an eigenfunction $u$ which does not change sign. As usual, we choose $u(\mathbf{x})>0$ and normalize it in $L^{2}$ on $\Omega$ (respectively on $\Omega \backslash B$ in the case of a hard obstacle).

Of particular interest for the the light they shed on the relationship of geomet ry to the fundamental eigenvalue are the following questions about the placement of the in terior obstacle:

1. Is it true that the optimal placement of an obstacle so as to minimize $\lambda$ is in contact with the boundary, while the the optimal placement to maximize $\lambda$ is in the interior?

2. Given an affirmative answer to Question 1, can the optimal position be located precisely?

For the placement of wells, we pose the same questions, with our expectations regarding the minimizing and maximizing positions reversed. In this article we shall describe some circumstances when the first question can be answered affirmatively, and so me more narrow circumstances when the second question can be answered.

These expectations are suggested by perturbative analysis. If either $\alpha \mathrm{o} r$ the size of $B$ is small, then to leading order in perturbation theory the effect of adding the a soft obstacle or well is asymptotic to

$$
\alpha \int_{B} u^{2} d^{n} x
$$

Thus (for example considering the case with $\alpha>0$ ) the strategy to minimize $\lambda$ is to place the obstacle near the boundary, while the strategy to maximize $\lambda$ is to place the obstacle in the interior, near the maximum of $u(x)$. The situation with a small hard obstacle is similar (using the estimates in [Fl95]), while the situation with a well is reversed.

In many of the situations in this article the inside region $B$ will be a ball. It is clear that for many purposes it is only necessary for $B$ to have certain reflection symmetries, but we have preferred to focus on the case where the statement of the result is simplest. (See Section IV, Example 7.)

In 1995, E.B. Davies asked two of us (E.H. and P.K.) questions of this type, for a hard spherical obstacle within a sphere. We answered the questions privately, using methods like those of this article: The minimizing position of the interior sphere is at the boundary of $\Omega$, while the maximizing position is at the center of the exterior $\Omega$. Quite recently we 
have learned from Mark Ashbaugh and Thierry Chatelain [As99] that in response to the same query from A.G. Ramm, they have answered it with similar methods.

Independently, one of us (K.K.) had been considering the problem of placing a positive potential with a specified integral within a region $\Omega$, so as to minimize $\lambda$. This work appears in [CGIKO99].

In both these independent lines of investigation, the minimizing obstacles are in contact with the boundary. One aim here is to explore this phenomenon further.

We are not aware of other work on this problem, although there are some asymptotic estimates for small obstacles [especially, F195], and some work on optimization of capacity on annular domains in [Fl93] and [Co94].

Our technique is to treat the motion of the obstacle or well as a perturbation, and estimate the perturbation with a reflection technique reminiscent of the classical method of moving planes [Al60 Se71, BeNi91], which, curiously, has heretofore not been used as much in spectral theory as in nonlinear analysis.

The first insight we use is that a translation of the obstacle or well can be regarded as a perturbation of a boundary. For a hard obstacle, the Hadamard boundary perturbation formula [Ha08, GaSc53i] applies. When specialized to the case of a translation, it reads simply:

Proposition I.1 (The Hadamard perturbation formula, special case).

Let $B$ be an interior hard obstacle, which can be moved rigidly a positive distance in the direction of a unit vector $\mathbf{v}$. The boundary of $B$ is assumed piecewise smooth. Then

$$
\frac{\partial \lambda}{\partial \mathbf{v}}=\int_{\partial B}|\nabla u|^{2} \mathbf{n} \cdot \mathbf{v} d S
$$

Here and throughout, $\mathbf{n}$ is the unit normal at the surface of the obstacle $B$. Our choice of orientation is outward with respect to $B$ and hence inward with respect to $\Omega$. We recall for later purposes that at a boundary with zero Dirichlet conditions, the gradient of $u$ is parallel to the normal vector, provided the latter is defined.

When $\alpha>0$ is finite, the derivative of the eigenvalue with respect to a translation of a soft obstacle is obtainable from Green's theorem. As for Hadamard's formula, the derivative is proportional to a certain surface integral. As a first step in deriving the formula for this derivative, we prepare an estimate for the eigenvalue $\lambda(\epsilon)$ and $L^{2}$-normalized eigenfunction $u(\epsilon)$ of a soft obstacle $B(\epsilon)$ obtained by shifting $B$ by a distance $\epsilon$.

\section{Lemma I.2.}

(a) There exist constants $\epsilon_{0}>0$ and $C$, depending only on $\Omega$, such that

$$
|\lambda(\epsilon)-\lambda| \leq C \epsilon
$$


for every $0<\epsilon<\epsilon_{0}$.

(b) $\|u(\epsilon)-u\|_{L^{\infty}(\Omega)} \rightarrow 0$ as $\epsilon \rightarrow 0$.

Proof: According to the min-max principle,

$$
\lambda(\epsilon) \leq \int_{\Omega}|\nabla u|^{2} d x+\alpha \int_{\Omega} \chi_{B(\epsilon)} u^{2} d x=\lambda+\alpha \int_{\Omega}\left(\chi_{B(\epsilon)}-\chi_{B}\right) u^{2} d x .
$$

Recall that $u$ and $u(\epsilon) \in L^{\infty}(\Omega)$ with $\|u(\epsilon)\|_{\infty} \leq C_{1}$, with $C_{1}$ depending only on $\Omega$ [see, e.g., GiTr83, Theorem 8.15]. Since also

$$
\int_{\Omega}\left|\left(\chi_{B(\epsilon)}-\chi_{B}\right)\right| d x \leq C_{2} \epsilon
$$

we obtain

$$
\lambda(\epsilon)-\lambda \leq C_{3} \epsilon
$$

By an analogous argument, $\lambda-\lambda(\epsilon) \leq C_{4} \epsilon$, completing the proof of (a).

Next, it is easy to see that $\{u(\epsilon)\}_{0<\epsilon<\epsilon_{0}}$ is bounded in $H_{0}^{1}(\Omega)$, so there exists a subsequence of $\{u(\epsilon)\}_{0<\epsilon<\epsilon_{0}}$ which converges weakly in $H_{0}^{1}(\Omega)$. Actually, $u(\epsilon)$ must converge to $u$ weakly in $H_{0}^{1}(\Omega)$ and strongly in $L^{2}(\Omega)$, since the weak limit of any subsequence of $\{u(\epsilon)\}_{0<\epsilon<\epsilon_{0}}$ is the first eigenfunction $u$ associated to $\lambda$ and hence unique. Let $w(\epsilon):=u(\epsilon)-u$. Then $w(\epsilon)$ satisfies

$$
-\Delta w(\epsilon)+\left(\alpha \chi_{B(\epsilon)}-\lambda\right) w(\epsilon)=(\lambda(\epsilon)-\lambda) u(\epsilon)-\alpha\left(\chi_{B(\epsilon)}-\chi_{B}\right) u .
$$

Applying the uniform $L^{\infty}$ estimate to $w(\epsilon)$, Applying the uniform $L^{\infty}$ estimate from [GiTr83, Theorem 8.15] to $w(\epsilon)$, we have

$$
\|w(\epsilon)\|_{L^{\infty}(\Omega)} \leq\|w(\epsilon)\|_{L^{2}(\Omega)}+C_{5}|\lambda(\epsilon)-\lambda|+C_{6}\left\|\chi_{B(\epsilon)}-\chi_{B}\right\|_{L^{1}(\Omega)} .
$$

This yields the desired estimate.

We shall need the following elementary formula:

\section{Lemma I.3.}

Suppose $\zeta \in C^{1}(\Omega)$. Then:

$$
\frac{1}{\epsilon}\left(\int_{B(\epsilon)} \zeta^{2} d x-\int_{B} \zeta^{2} d x\right) \rightarrow \int_{\partial B} \zeta^{2}(\mathbf{v} \cdot \mathbf{n}) d S
$$

as $\epsilon \rightarrow 0$.

Proof: Since $\int_{B(\epsilon)} \zeta^{2} d x=\int_{B} \zeta^{2}(\mathbf{y}+\epsilon \mathbf{v}) d y$, we get

$$
\frac{1}{\epsilon}\left(\int_{B(\epsilon)} \zeta^{2} d x-\int_{B} \zeta^{2} d x\right)=\int_{B(\epsilon)}\left(\frac{\zeta(\mathbf{y}+\epsilon \mathbf{v})-\zeta(\mathbf{y})}{\epsilon}\right)(\zeta(\mathbf{y}+\epsilon(\mathbf{v}))+\zeta(\mathbf{y})) d y
$$




$$
\rightarrow 2 \int_{B} \frac{\partial \zeta}{\partial \mathbf{v}} \zeta d y=\int_{B} \nabla \cdot\left(\zeta^{2} \mathbf{v}\right) d y .
$$

The divergence theorem implies the desired result.

\section{Proposition I.4.}

Consider the case of a soft obstacle or a well (1.1), where B is assumed to have a piecewise smooth boundary. Suppose that B can be moved rigidly a positive distance in the direction of a unit vector $\mathbf{v}$. Then

$$
\frac{\partial \lambda}{\partial \mathbf{v}}=\alpha \int_{\partial B}|u|^{2} \mathbf{n} \cdot \mathbf{v} d S .
$$

Proof: We denote by $\lambda(\epsilon)$ and $\lambda$ the fundamental eigenvalues of the operators $-\Delta+\alpha \chi_{B(\epsilon)}$ and $-\Delta+\alpha \chi_{B}$, respectively. Here $B(\epsilon)=\left\{\mathbf{y} \in \mathbf{R}^{n} ; \mathbf{y}=\mathbf{x}+\epsilon \mathbf{v}\right\}$ for small $\epsilon>0$. We choose $0<\epsilon<\epsilon_{0}$, where $\epsilon_{0}>0$ is sufficiently small that $B(\epsilon) \subset \Omega$. We also denote by $u(\epsilon)$ and $u$ the $L^{2}$-normalized eigenfuctions associated with $\lambda(\epsilon)$ and $\lambda$, respectively. Thus $u(\epsilon)$ and $u$ satisfy

$$
\int_{\Omega} \nabla u(\epsilon) \cdot \nabla \phi+\alpha \chi_{B(\epsilon)} u(\epsilon) \phi d x=\lambda(\epsilon) \int_{\Omega} u(\epsilon) \phi d x
$$

and

$$
\int_{\Omega} \nabla u \cdot \nabla \psi+\alpha \chi_{B} u \psi d x=\lambda \int_{\Omega} u \psi d x
$$

for every $\phi, \psi \in H_{0}^{1}(\Omega)$. Substituting $\phi=u$ and $\psi=u(\epsilon)$, we have

$$
(\lambda(\epsilon)-\lambda) \int_{\Omega} u(\epsilon) u d x=\alpha \int_{\Omega}\left(\chi_{B(\epsilon)}-\chi_{B}\right) u(\epsilon) u d x .
$$

By Lemma I.2 (b), we have

$$
\left|\int_{\Omega} u(\epsilon) u d x-\int_{\Omega} u^{2} d x\right|=o(1),
$$

and

$$
\left|\int_{\Omega}\left(\chi_{B(\epsilon)}-\chi_{B}\right)\left(u(\epsilon) u-u^{2}\right) d x\right|=o(\epsilon) .
$$

Hence it follows from Lemma I.2 (a) that

$$
\frac{(\lambda(\epsilon)-\lambda)}{\epsilon}=\frac{\alpha}{\epsilon} \int_{\Omega}\left(\chi_{B(\epsilon)}-\chi_{B}\right) u^{2} d x+o(1) .
$$

Since $u \in C^{1, \beta}(\Omega)$ for $0<\beta<1$ (see, e.g., [GiTr83]), Lemma I.3 yields

$$
\frac{d \lambda}{d \mathbf{v}}=\left.\frac{d \lambda(\epsilon)}{d \epsilon}\right|_{\epsilon=0}=\alpha \int_{\partial B} u^{2}(\mathbf{v} \cdot \mathbf{n}) d S .
$$


Since the possible centers of the obstacle or well form a compact subset of $\Omega$, it is immediate from Lemma I.1 and Lemma I.2 that under the assumptions of this article:

The maximizing and minimizing positions of $B$ exist.

\section{The technique of domain reflection}

If a domain has a certain reflection property with respect to an axis of symmetry of the obstacle, then we shall be able to identify the sign of the directional derivative of the fundamental eigenvalue with respect to the position of an obstacle or well. Roughly speaking, when this property holds, we shall show that the eigenvalue increases as the obstacle moves away from a nearby portion of the boundary of $\Omega$.

To avoid complications, we henceforth assume that the set $B$ is convex as well as piecewise smooth. We also require that it be reflection-symmetric about some hyperplane (or plane, or line) $P$ of dimension $n-1$. When we consider specific examples, $B$ will often be a ball.

Definition. Let $P$ be a hyperplane of dimension $n-1$ which intersects $\Omega$. For any connected set $S$ which does not intersect $P$, we let $S^{P}$ denote its reflection through $P$. The domain $\Omega$ is said to have the interior reflection property with respect to $P$ if there is a connected component $\Omega_{s}$ of $\Omega \backslash P$ such that $\Omega_{s}^{P}$ is a proper subset of the other connected component $\Omega_{b}$ of $\Omega \backslash P$. Any such $P$ will be called a hyperplane of interior reflection for $\Omega$. Moreover, $\Omega_{s}$ will be called the small side of $\Omega$ (and $\Omega_{b}$ the big side).

The following theorem states formally that when this property holds, the eigenvalue is strictly increasing as a symmetric obstacle is moved away from the small side:

\section{Theorem II.1.}

Assume that $\Omega$ has the interior reflection property with respect to a hyperplane $P$ about which the set $B$ is reflection-symmetric. Suppose that $B$ is translated in the direction of a unit vector $\mathbf{v}$ perpendicular to $P$ and pointing from the small side to the big side.

Then, in the case of a hard or soft obstacle,

$$
\frac{d \lambda}{d \mathbf{v}}>0
$$

In the case of a well,

$$
\frac{d \lambda}{d \mathbf{v}}<0
$$

\section{Remark.}

Actually, the soft obstacle or well here could be any reflection-symmetric function supported within the closure of $B$, not just its indicator function. 
Proof: There are three cases to consider, that of a hard obstacle, a soft obstacle, and a well. We consider the hard obstacle last.

For the other two cases, we claim that for any point $\mathbf{x}$ of $\partial B$ which is on the small side of $\Omega, u(\mathbf{x})<u\left(\mathbf{x}^{P}\right)$. The theorem will then follow in these cases from (1.3).

To establish the claim, we consider $w(\mathbf{x}):=u(\mathbf{x})-u\left(\mathbf{x}^{P}\right)$ on the small side $\Omega_{s}$. On the interior of this region,

$$
\left(-\nabla^{2}+\alpha \chi_{B}\right) w=\lambda w
$$

while on its boundary, $w(\mathbf{x}) \leq 0$. Observing that $w$ is strictly negative on part of that boundary and that $\lambda$ is less than the fundamental Dirichlet eigenvalue of $-\nabla^{2}+\alpha \chi_{B}$, we conclude from the maximum principle [PrWe84] that $w(\mathbf{x})<0$ in the interior of this region, and hence that $u(\mathbf{x})<u\left(\mathbf{x}^{P}\right)$ for $\mathbf{x}$ in $\partial B$ on the small side of $\Omega$.

This establishes the claim except for the case of a hard obstacle, where we use the Hadamard formula (1.2) in place of (1.3). This time we consider the function $w(\mathbf{x}):=$ $u(\mathbf{x})-u\left(\mathbf{x}^{P}\right)$ on the small side $\Omega_{s}$ but excluding $B$. Just as before, the maximum principle tells us that $w(\mathbf{x})<0$ on the interior of this region. To finish the proof in this case, we appeal to the boundary-point lemma of [Se71, p. 308], according to which, at every smooth point of the part of $\partial B$ on the small side, either the normal derivative of $w(\mathbf{x})$ is strictly positive, or else the second derivative of $w(\mathbf{x})$ in this direction is strictly positive. However, the latter possibility is excluded because it contradicts the eigenvalue equation (since the Laplacian of $w$ is negative while all second derivatives in tangential directions at the boundary are 0$)$. Hence $|\nabla u(\mathbf{x})|<\left|\nabla u\left(\mathbf{x}^{P}\right)\right|$ for $\mathbf{x}$ in $\partial B$ on the small side of $\Omega$. The theorem then follows from (1.2).

In the final section of this article we consider many examples where it can be shown that either the domain $\Omega$ or a suitable related domain $\Omega^{\prime}$ contains a dense subset of points which lie on a hyperplane of interior reflection. In preparation for that we state here an obvious corollary of Theorem II.1:

\section{Corollary II.2.}

Let $\mathbf{x} \in \Omega$ denote the center of a spherical obstacle B. At any maximizing $\mathbf{x}$,

(a) $\Omega$ has no hyperplane $P$ of interior reflection containing $\mathbf{x}$.

Moreover, at any minimizing $\mathbf{x}$, either statement a) above is true, or else

(b) $\partial B$ intersects the small side of $\partial \Omega$.

Convexity ensures that $\Omega$ enjoys the interior reflection property with respect to some secant plane passing through any point sufficiently close to the boundary. This immediately implies:

\section{Corollary II.3.}

Assume that $\Omega$ is convex and that $B$ is a ball of radius $\rho$. There exists $R_{0}>0$ depending on $\Omega$ such that if $\rho<R_{0}$, then there are neighborhoods $N_{1,2}$ of the boundary, such that 
(a) The maximizing (resp., minimizing) obstacle (resp., well) for $\lambda$ lies outside $N_{1}$; and

(b) Any obstacle (resp., well) which minimizes (resp., maximizes) $\lambda$ subject to being located within $N_{2}$; must touch the boundary of $\Omega$

In principle, given any convex $\Omega$ it is straightforward to identify neighborhoods $N_{1,2}$ explicitly. In the following section we consider some cases where $N_{2}=\Omega$ and where the optimal positions can be determined exactly, sometimes even without convexity.

At the level of generality of Corollary II. 3 there is a "hole" in the interior of a convex $\Omega$ within which we can say little about the optimal placement of obstacles. With a reflection symmetry, however, the hole can be reduced to a slit, because together with convexity this implies that every point of $\Omega$ is either reflection-symmetric or else on a hyperplane of interior reflection:

\section{Corollary II.4.}

Suppose $\Omega$ and the obstacle are as in Corollary II.3, and in addition that $\Omega$ is symmetric with respect to reflection through a hyperplane $H$. Then at the minimizing position the obstacle is in contact with the boundary, while at the maximizing position its center is on $H$.

Next we note an extension of Theorem II.1 to the case of Schrödinger operators, which is useful for discussions of soft obstacles and of interest in its own right.

\section{Theorem II.5.}

Consider the Schrödinger operator $\mathcal{H}:=-\nabla^{2}+V(\mathbf{x})$ on a domain $\Omega$, where the potential $V(\mathbf{x})$ is a real-valued function in $L^{\infty}(\Omega)$ (or more generally satisfying conditions guaranteeing that the fundamental eigenvalue is discrete; see, e.g. [ReSi78]). Assume that $\Omega$ has the interior reflection property with respect to a hyperplane $P$ about which the set $B$ is reflection-symmetric, and that on the small side $\Omega_{s}$,

$$
V(\mathbf{x}) \geq V\left(\mathbf{x}^{P}\right) \text { a.e. }
$$

Suppose that $B$ is translated in the direction of a unit vector $\mathbf{v}$ perpendicular to $P$ and pointing from the small side to the big side.

Then, in the case of a hard or soft obstacle (i.e., $\left.-\nabla^{2}+V(\mathbf{x})+\alpha \chi_{\mathrm{B}}(\mathbf{x})\right)$,

$$
\frac{d \lambda}{d \mathbf{v}}>0
$$

In the case of a well,

$$
\frac{d \lambda}{d \mathbf{v}}<0
$$


Proof: As for Theorem II.1. This time, however, $w(\mathbf{x}):=u(\mathbf{x})-u\left(\mathbf{x}^{P}\right)$ is no longer a solution of the eigenvalue equation on $\Omega_{s}$ but is instead a subsolution, i.e.,

$$
(H-\lambda) w(\mathbf{x})=-\left(V(\mathbf{x})-V\left(\mathbf{x}^{P}\right)\right) u\left(\mathbf{x}^{P}\right) \leq 0 .
$$

This, however, suffices for the maximum principle, by the following argument:

First we observe that $w(x) \leq 0$ on $\Omega_{s}$ as before. Indeed, if $U=\{w(x)>0\}$ were non-empty, then the inequality in the proof of Theorem II.5 would imply that $\lambda \geq \mu_{1}$, where $\mu_{1}$ is the first eigenvalue of $H$ on $U$. Yet $U \subset \Omega_{s} \subset \Omega$ and $\lambda$ is the first eigenvalue of $H$ on $\Omega$. As a consequence of the unique continuation theorem [e.g., JeKe85], we find that $\mu_{1}>\lambda$, which is a contradiction.

Next, to conclude that $w(x)<0$ in $\Omega_{s}$, we appeal to the strong maximum principle.

We close this section by observing that for sufficiently small $\alpha$, any globally minimizing soft obstacle (resp., maximizing well) touches the boundary:

\section{Theorem II.6.}

Suppose $\Omega$ is convex and that it contains a soft spherical obstacle, i.e., a potential $\alpha \chi_{B}(x)$, where $B$ is a sufficiently small ball. Then there exists $\alpha_{0}>0$ such that for every $\alpha$ with $0<\alpha<\alpha_{0}$, when $B$ is at the position where it minimizes the first eigenvalue, it touches the boundary $\partial \Omega$.

Proof: We let $B_{\epsilon}(\mathbf{w})$ denote the obstacle when centered at $\mathbf{w}$, and we ssume that the radius $\epsilon>0$ of the obstacle is sufficiently small.

First we claim that there exists a compact subset $G \subset \Omega$, independent of $\epsilon$, such that if $\mathbf{w} \in G^{\prime}$ and $\overline{B(\mathbf{w})} \subset \Omega$, then $B(\mathbf{w})$ cannot be an optimal obstacle. Here $G^{\prime}:=\Omega \backslash G$. This follows from Corollary II.3. We observe that $G$ may be chosen independently of $\epsilon$ for $\epsilon$ sufficiently small; the choice of $G$ depends only on $\Omega$.

Let $\psi$ be the $L^{2}$ normalized first eigenfunction of the Dirichlet Laplacian on $\Omega$, and let $[\Omega]^{\delta}=\{x \in \Omega ; \psi(x)>\delta\}$ for $\delta>0$. Then there exists a small $\delta>0$ such that $G \subset \subset[\Omega]^{\delta}$. We fix a value of the radius $\epsilon>0$ sufficiently small so that

$$
\epsilon<\min \left(\operatorname{dist}\left(\partial G, \partial[\Omega]^{\delta}\right),(1 / 2) \operatorname{dist}\left(\partial \Omega, \partial[\Omega]^{\delta}\right)\right)
$$

Next we assert that, as shown in [CGIKO99], there exists a small $\alpha_{0}>0$ such that if $\mathbf{w} \in G$, then $B_{\epsilon}(\mathbf{w})$ cannot be a minimizing obstacle for any $0<\alpha<\alpha_{0}$, because

$$
\|u(\epsilon, \mathbf{w})-\psi\|_{L^{\infty}(\Omega)} \leq C \alpha
$$

for some constant $C$ which does not depend on w. 
Here $u(\epsilon, \mathbf{w})$ denotes the $L^{2}$ normalized first eigenfunction of $-\Delta+\alpha \chi_{B_{\epsilon}(\mathbf{w})}$. Note that if $\mathbf{w} \in G$, then $B_{\epsilon}(\mathbf{w}) \subset\{\psi(x)>\delta\}$, and we can find a ball $B_{\epsilon}\left(\mathbf{w}^{\prime}\right) \subset\{\psi(x)<\delta\}$. Hence, for sufficiently small $\alpha_{0}$,

$$
\int_{B_{\epsilon}(\mathbf{w})} u(\epsilon, \mathbf{w})^{2} d x>\int_{B_{\epsilon}\left(\mathbf{w}^{\prime}\right)} u(\epsilon, \mathbf{w})^{2} d x
$$

for all $0<\alpha<\alpha_{0}$. Together with the variational principle, this implies that $B_{\epsilon}(\mathbf{w})$ cannot be an minimizing obstacle for $\mathbf{w} \in G$ and for $0<\alpha<\alpha_{0}$, completing the proof.

\section{Optimization at a vertex or corner}

It is not difficult to show that an ellipse has the interior reflection property with respect to any secant line which is perpendicular to the boundary at one of its crossing points and which does not coincide with one of the axes. It thus follows fairly easily from Theorem II.1 that if the radius of the ball $B$ is sufficiently small so that it fits inside an elliptical domain $\Omega$, then the minimizing ball touches the boundary. Actually, we can locate the minimizing position at the vertex of the ellipse, and the maximizing position at the center, for a class of domains generalizing the ellipse (see Theorem III.2, below).

We shall show that this phenomenon, that minimizing obstacles are located at parts of the boundary where the curvature is maximized, also occurs in certain other situations. Unfortunately, we are not able to determine the degree of generality of this phenomenon.

We begin by extending Theorem II.1 to the case where $B$ moves along the boundary; To keep the statement simple, we restrict to the case of spherical $B$.

\section{Proposition III.1.}

Let $B$ be a ball which is tangent to the boundary of $\Omega$, assumed of class $C^{2}$ in a neighborhood of the point of contact. Suppose furthermore that $\Omega$ has the interior reflection property with respect to a hyperplane $P$ normal to the boundary at the point of contact. Then $\lambda$ is strictly increasing as $B$ is moved in contact with the boundary towards the big side.

Proof sketch: The argument is by domain perturbation as for Theorem II.1, with the further complication that as the domain $B$ moves along a smooth boundary, it is not only translated and but also continuously rotated. For non-spherical domains, Propositions I.1 and I.4 would need to be modified with additional terms to reflect this. For spherical domains, however, the additional terms do not arise, and the formulae for the directional deivatives are as before.

We next identify a class of roughly elliptical regions for which we can carry out a complete analysis of the maximizing and minimizing positions of an obstacle or well.

Definition. A vertex of a domain with boundary of class $C^{2}$ is a point on the bounda ry at which the curvature is locally maximal. Outward pointing corners of a piecewise $C^{2}$ boundary are also considered vertices. 


\section{Theorem III.2.}

Let $\Omega$ be a two-dimensional convex domain with the following properties:

(a) $\Omega$ is reflection symmetric with respect to both the $x$ and $y$ Cartesian axes.

(b) The boundary of $\Omega$ is of class $C^{2}$ for $x, y \neq 0$.

(c) In any quadrant of the plane, the curvature of the boundary of $\Omega$ is monotonic as a function of $x$ (or equivalently of $y$, or equivalently of the arclength $s$ ).

Suppose that the obstacle (respectively, well) $B$ is a disk.

If the radius of $B$ is less than the radius of curvature at the vertex of $\Omega$, then $\lambda$ is minimized (resp. maximized) when the $B$ is in contact with a vertex, and maximized (resp. minimized) when the obstacle (resp. well) is at the origin.

This theorem certainly generalizes to three-dimensional ellipsoidal domains $\Omega$ which are rotationally symmetric. We also remark that, as a special case, when both $\Omega$ and $B$ are balls, $\lambda$ is a strictly increasing function of the distance of $B$ from the boundary until it reaches the center, where it is maximized. (This answers the query of Davies, 1995.)

Theorem III.2. is a direct corollary of:

\section{Proposition III.3.}

A region as in Theorem III. 2 enjoys the interior reflection property with respect to any line normal to its boundary, except for the lines of symmetry ( $x$ and $y$ axes). The small side of the normal line at a boundary point $P$ is the side of increasing curvature of the boundary moving from $p$.

\section{Proof:}

We may and shall assume without loss of generality that the curvature of the boundary of $\Omega$ is strictly increasing as a function of $y$ in the first quadrant of the plane (and hence strictly monotonic in any quadrant of the plane). We also orient the arclength $s$ counterclockwise, so that $s$ is an increasing function of $y$. We observe that $\Omega$ is convex and that in the first quadrant the distance from the origin to a point on $\partial \Omega$ increases with $y$. For simplicity we assume that $\partial \Omega$ is of class $C^{2}$ even at the possibly exceptional points $(x=0$ or $y=0)$; it will be clear that this does not affect the result.

Let $\varphi$ be the angle of the normal to the boundary as measured counterclockwise from the $x$-axis. Because of our assumptions, the angle $\varphi$ can be used to parametrize the points of $\partial \Omega$; we henceforth do this, and use the notation $P(\varphi)$ for those points. Let $L$ designate the normal line to $\partial \Omega$ at some specific $P(\alpha)$ in the first quadrant.

We claim first that if $\beta:=$ the angle $>\alpha$ where $L$ intersects $\partial \Omega$, then $\beta<\pi+\alpha$. The statement that $\beta<\pi+\alpha$ is easily seen to be equivalent to the statement that $L$ passes above the center of $\Omega$, which in turn means that $\varphi<\theta$, where $\theta$ is the usual polar coordinate of $P(\alpha)$.

In order to show that the reflection of $\partial \Omega$ for $\alpha<\varphi_{+}<\beta$ fits within $\Omega$, we make some definitions. Let $\ell_{+}$be the distance from $P\left(\varphi_{+}\right)$to $L$ and $t$ as the distance from $P(\alpha)$ 
to to the point on $L$ closest to $P\left(\varphi_{+}\right)$. At a given value of $t$, there is an analogous point $P\left(\varphi_{-}\right)$on the other side of $L$, at some $\varphi_{-}<\alpha$; we define the distance from $P\left(\varphi_{-}\right)$to $L$ as $\ell_{-}$

We thus need to show that $\ell_{+} \leq \ell_{-}$for the same value of $t$. Hence we consider the maps $\varphi_{ \pm} \rightarrow t$, for which

$$
d t=\sin (\varphi-\alpha) d s=\frac{1}{\kappa(\varphi)}|\sin (\varphi-\alpha)| d \varphi
$$

Here, $\kappa$ designates the curvature of the boundary at the point corresponding to $\varphi$. For $\alpha<\phi<\alpha+\frac{\pi}{2}$

$$
\begin{aligned}
t(\varphi) & -t(2 \alpha-\varphi) \\
& =\int_{\alpha}^{\varphi}\left(\frac{1}{k(\phi)}-\frac{1}{k(2 \alpha-\phi)}\right)|\sin (\phi-\alpha)| d \phi \\
& <0 \quad \text { for } \theta<\alpha+\frac{\pi}{2},
\end{aligned}
$$

since the integrand is negative

Furthermore, this difference is negative for all $\alpha<\varphi<\beta$ becaus e the integral is antisymmetric about $\frac{\pi}{2}+\alpha$ and $\beta<\left(\frac{\pi}{2}+\alpha\right)+\left(\frac{\pi}{2}-\alpha\right)=\pi$. We conclude that $t(\varphi)>$ $t(2 \alpha-\varphi)(2 \alpha-\varphi$ is the angle reflected through $\alpha)$ for this range of $\varphi$, and therefore if we consider the two maps $\varphi \rightarrow t$, we see:

$$
|\varphi-\alpha|(t)_{\text {aboveL }}>|\varphi-\alpha|(t)_{\text {below } L}
$$

Also: both $t(\varphi)$ and $t(2 \alpha-\varphi)$ are monotonic increasing functions of $\varphi$ for $\varphi>\alpha$.

Since $\frac{d \ell_{ \pm}}{d s}=\cot (\varphi-\alpha)$ and cot is a decreasing function for $0<|\varphi-\alpha|<|\beta-\alpha|<\pi$, we conclude by integrating that

$$
\ell_{+}<\ell_{-} \quad \text { for } 0<t<t(\beta)
$$

\section{Theorem III.4.}

(a) Suppose that $\Omega$ and $B$ are as in Theorem III.2, and that the radius of $B$ is small enough for it to fit within $\Omega$ but larger than the radius of curvature at its vertex. Then $\lambda$ is minimized (resp. maximized) when $B$ is in as close as possible to a vertex, and maximized (resp. minimized) when the obstacle (resp. well) is at the origin.

(b) Suppose that $\Omega$ is an equilateral polygon centered at the origin. Then $\lambda$ is minimized (resp. maximized) when the ball is as close as possible to any vertex of $\Omega$, and maximized (resp. minimized) when the obstacle (resp. well) is at the origin. 
Proof: We discuss the case of an obstacle. As usual, the case of a well uses the same argument, with a reversal of "maximal" and "minimal".

Part a) is an obvious variant of Theorem III.2. It is only necessary to notice that if $B$ is anywhere other than at the origin, the interior reflection holds with respect to the horizontal or vertical plane through the center of $B$. By Corollary II.1, the only possibility for the maximizing position is the center, whereas at the minimizing position $B$ is in contact with $\partial \Omega$.

To localize the minimizer more precisely, we observe that if the contact point is not a point of symmetry of $\partial \Omega$, and $B$ is not obstructed from displacements to the small side of $\Omega$, then Proposition III.1 excludes the configuration as a candidate for a minimizer. It is moreover easy to see that any attainable point of symmetry other than a vertex is excluded from being a contact point of a minimizing obstacle. (It would in fact be a constrained maximizing position, given contact with the boundary.) The only remaining possibility is that described in statement a).

The argument for part b) is similar. From Theorem II.1, using the symmetry of the polygon we see that if the center of $B$ is anywhere other than at the center of $\Omega$, then it lies on a hyperplane of interior reflection, and $\lambda$ decreases as $B$ moves perpendicularly away from any line of symmetry of $\Omega$. The argument at the boundary is much as for case a). The perpendicular line from the point of contact is a hyperplane of interior reflection except when the contact is at the midpoint of an edge of the polygon, but $\lambda$ decreases when the point of contact is moved away from a midpoint on either side. The claim then results from possibility b) of Corollary II.2.

\section{Extensions, and some instructive examples}

Although at first sight our technique seems to be restricted to regular, convex regions, we now proceed to illustrate by example how it can be extended. In this section we describe only problems with obstacles. In most cases, however, the same examples illustrate the situation for wells, with the usual reversal of "min" and "max."

We shall omit details of the proofs when they consist only in recalling Corollary II.2 and elementary exercises in finding possible hyperplanes of interior reflection.

\section{Example 1.}

Let $\Omega$ be a finite region bounded by two spheres in $R^{N}$, and suppose that a spherical obstacle $B$ has radius $\rho$ small enough so that it fits into $\Omega$. For definiteness, suppose that the larger sphere has its center at the origin and radius $R$, and that the smaller one has its center on the $x_{1}$ axis at coordinate $x_{1}=a \geq 0$, and radius $r \leq R$.

There are five possibilities:

(a) $\Omega$ is gibbous (simply connected and convex: $\Omega$ is the intersection of two balls, and $R-r<a<R+r)$. Then at the minimizing positions of $B$, it is as near as possible 
to a vertex. (There are two such positions if $N=2$, and otherwise they form a sphere of dimension $N-2$.) At the maximizing position $\mathbf{x}$ the center of $B$ is located on the $x_{1}$ axis with $x_{1}$ in the interval $\left[\frac{R-r+a}{2}, \frac{R^{2}-r^{2}+a^{2}}{2 a}\right] \cap[a-r+\rho, R-\rho]$. (Note: $\frac{R^{2}-r^{2}+a^{2}}{2 a}$ is the $x$-coordinate of the intersection of the two circles.)

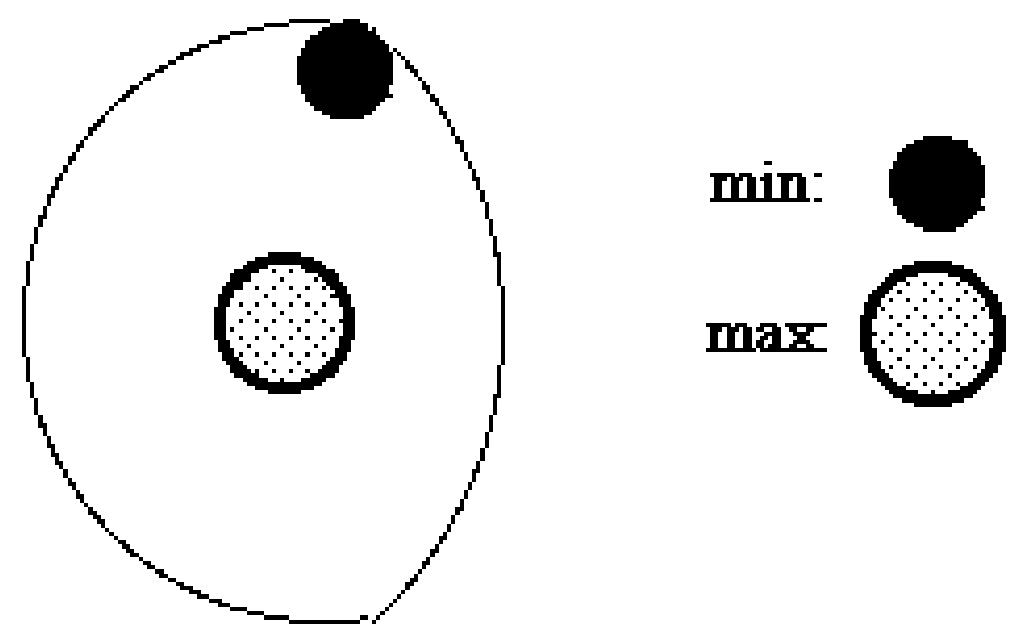

\section{Example 1(a)}

(b) $\Omega$ is crescent (simply connected and nonconvex: $\Omega$ is the intersection of the larger ball and the exterior of the smaller ball, and $R-r<a<R+r)$. Then at the minimizing positions of $B$, it is as near as possible to a vertex. (There are two such positions if $N=2$, and otherwise they form a sphere of dimension $N-2$.) At the maximizing position $\mathbf{x}$ the center of $B$ is located on the $x_{1}$ axis with $x_{1}$ in the interval $\left[\frac{-R-r+a}{2}, 0\right] \cap[-R+\rho, a-r-\rho]$.

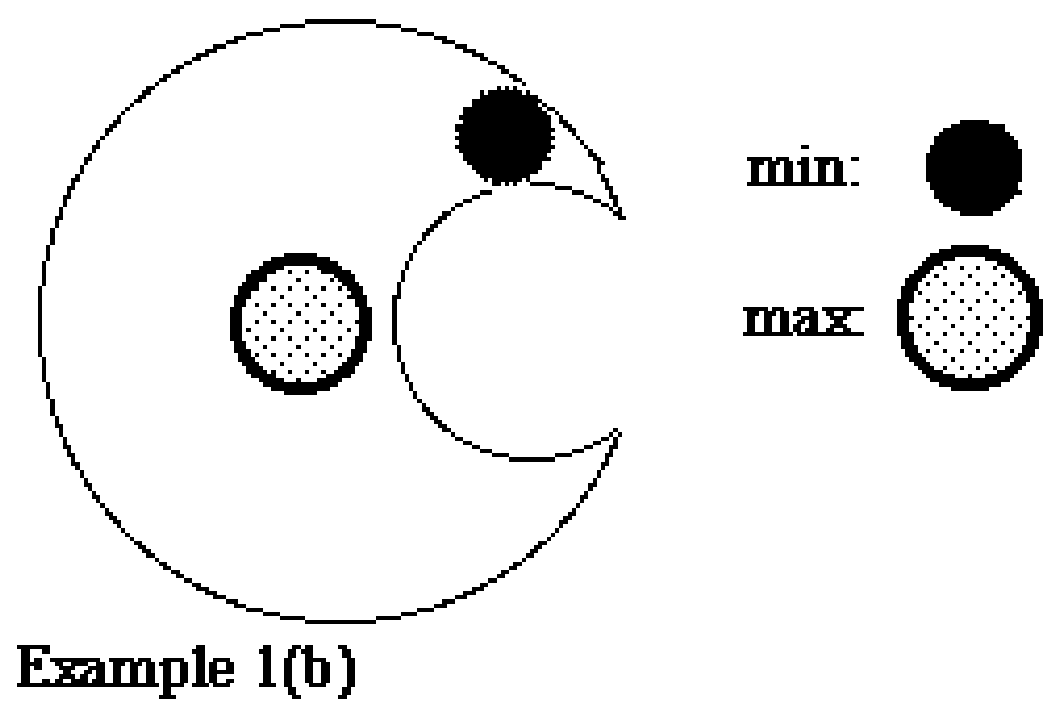

(c) $\Omega$ is annular but not concentrically so, and $\rho>\frac{R-(r+a)}{2}$. This is essentially like the case of the crescent: At the minimizing positions of $B$, it is as near as possible to the point $x_{1}=R$ on the $x_{1}$-axis. (There are two such positions if $N=2$, and otherwise they 
form a sphere of dimension $N-2$.) The maximizing position $\mathbf{x}$ of the center of $B$ is located on $x_{1}$ axis with $x_{1}$ in the interval $\left[\frac{-R-r+a}{2}, 0\right] \cap[-R+\rho, a-r-\rho]$, as in part $(\mathrm{b})$.

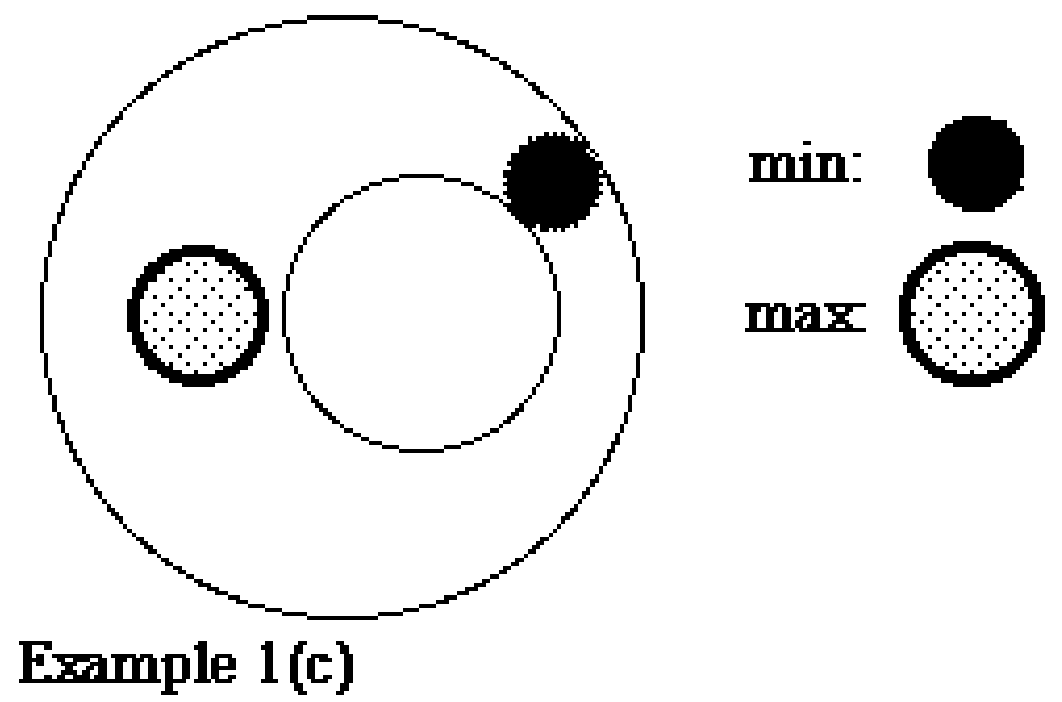

(d) $\Omega$ is annular but not concentrically so, and $\rho<\frac{R-(r+a)}{2}$. The maximizing position $\mathbf{x}$ of $B$ is as in parts (b) and (c). The minimizing positions satisfy $a+r+\rho<x_{1}<\frac{a+R+r}{2}$.

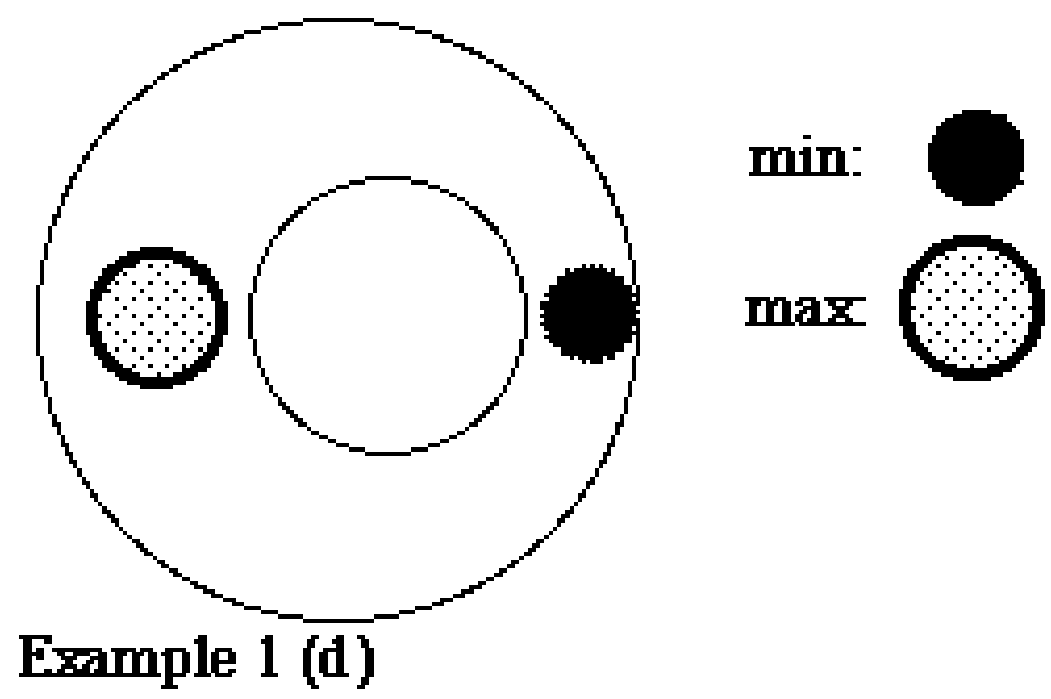

(e) $\Omega$ is a concentric annulus (spherical shell). Necessarily, $\rho<\frac{R-r}{2}$. At maximum, the position of the center $\mathbf{x}$ of $B$ satisfies $r+\rho \leq|\mathbf{x}| \leq \frac{R+r}{2}$. At minimum, either $\mathbf{x}$ is in the same annulus or else $|\mathbf{x}|=R-\rho$ (contact with the outer boundary).

These rely on Corollary II.2; among the hyperplanes of interior reflection to consider are horizontal hyperplanes and the bisecting planes of the spheres. Our technique does not allow us to eliminate one of the two possibilities for the minimum in 1 (e). We suspect the true alternative to be that the minimizing obstacle is in contact with the outer boundary, and will establish this in Example 12 when the inner radius is sufficiently small and the 
obstacle is soft.

\section{Example 2.}

Horseshoe-shaped domains: Let $\Omega_{0}$ be the concentric annular domain of Example 1(e) in 2 -dimensions, and let $S_{\alpha}$ be the sector $\left|x_{2}\right|<\alpha x_{1}$ for some $\alpha>0$. For some fixed opening angle $\beta \leq \pi / 2$, let $\sigma_{ \pm}$denote two open circular sectors of radius $R-r$ and centered at the inner edge of $\Omega_{0}$, i.e., at $\left(\frac{r}{\left(1+\alpha^{2}\right)^{1 / 2}}, \pm \frac{\alpha r}{\left(1+\alpha^{2}\right)^{1 / 2}}\right)$, such that one edge lies on $\partial S_{\alpha}$. $\Omega:=\left\{\Omega_{0} \cap S_{\alpha}^{c}\right\} \cup \sigma_{-} \cup \sigma_{+}$. The hard or soft obstacle is a disk of radius $<R-r$.

The maximizing position is on the $x_{1}$-axis and is otherwise as in Example 1 (e), while the unique two minimizing positions have the obstacle wedged in the corners of the sectors $\sigma_{ \pm} \cdot$

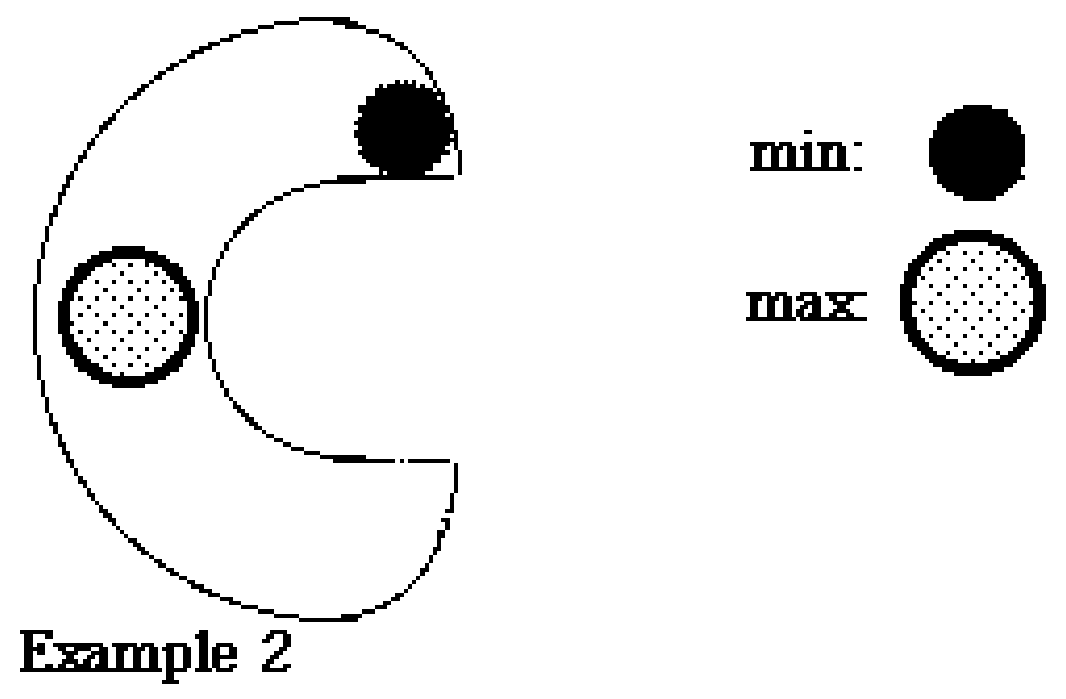

The proof is obtained by noting that rays from the origin other than the negative $x_{1}$-axis are lines of interior reflection whenever they intersect $\Omega_{0}$, as are rays beginning at $\left(\frac{r}{\left(1+\alpha^{2}\right)^{1 / 2}}, \pm \frac{\alpha r}{\left(1+\alpha^{2}\right)^{1 / 2}}\right)$, and passing through $\sigma_{ \pm}$.

We note that appending $\sigma_{ \pm}$allowed us to be precise about the minimizing position; without them, the minimizing position would be in contact with $\partial S_{\alpha}$, but our method would not give the exact position.

\section{Example 3.}

(a) A ball $\Omega$ with two interior hard spherical obstacles $B_{1}$ and $B_{2}$, which can be placed independently, and may have different radii, the sum of which is less than the radius of $\Omega$. In this case the minimizing configuration has both obstacles touching the boundary and each other. In the maximizing configuration the centers of $B_{1}$ and $B_{2}$ lie on a diameter of $\Omega$.

(b) The same example, except that one of the obstacles is allowed to be soft. The maximizing and minimizing configurations are as for part (a). 


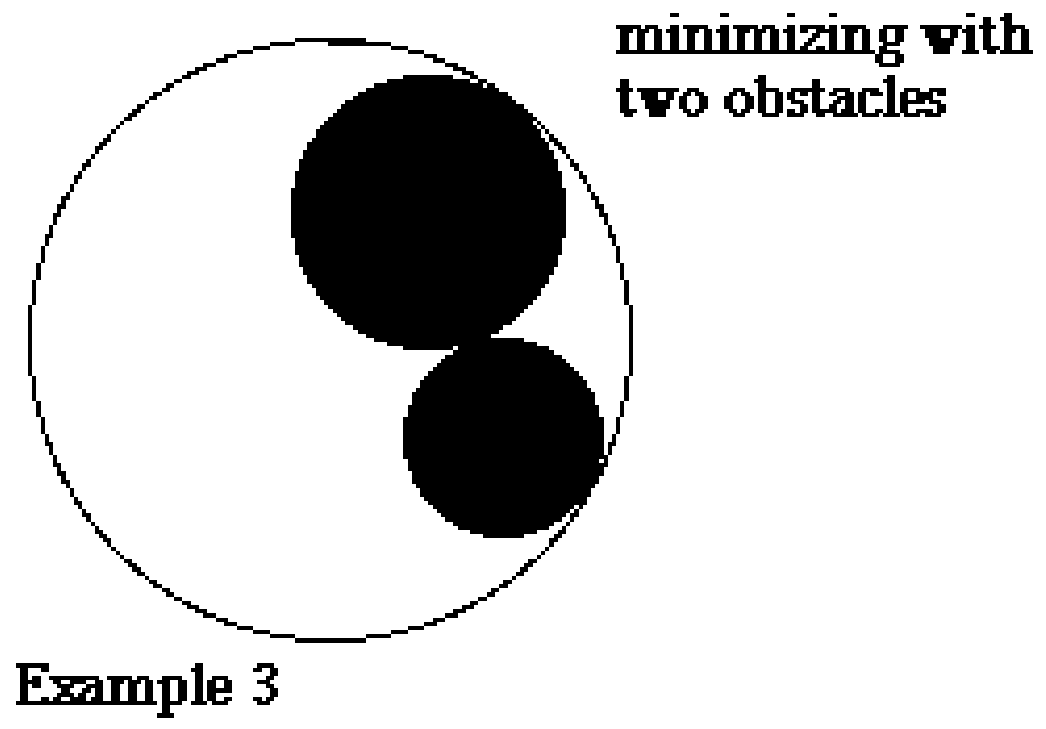

We observe that the minimizing configuration disconnects $\Omega$.

Denote by $\mathbf{x}_{1}$ and $\mathbf{x}_{\mathbf{2}}$ the centers of the balls $B_{1}$ and $B_{2}$, respectively. We begin by considering the relative positions of the balls as fixed a priori and treating them as a single obstacle. Unless they lie on a diameter of $\Omega$, the line passing through both their centers is a line of interior reflection. We thus conclude that at maximum they lie on a diameter of $\Omega$, while at minimum at least one of them touches $\partial \Omega$.

Having established that at least one obstacle touches the boundary when $\lambda_{1}$ is minimized, we can assume for the minimizing problem that one spherical obstacle is fixed to $\partial \Omega$ in some standard orientation, rotating the entire problem as necessary, while letting the position of the second obstacle vary. This, however, is the situation of Example 1 (b). Hence we know that at minimum the two obstacles touch each other and $\partial \Omega$.

\section{Example 4.}

(a) The same as Example 3, except that we also insert a third, hard obstacle of positive capacity and any shape small enough that it can be translated and rotated so as to fit inside $\Omega_{s}:=$ the smaller of the two domains into which $\Omega$ is disconnected at the minizing configuration of Example 3. ( $\Omega_{1}$ is of course only defined up to rotations of the two larger obstacles about the center of $\Omega$.) Then the minimizing configuration is as in Example 3 , with the third obstacle anywhere within $\Omega_{1}$. This can be a unique minimizer or highly nonunique, depending on the size and shape of the third obstacle. 


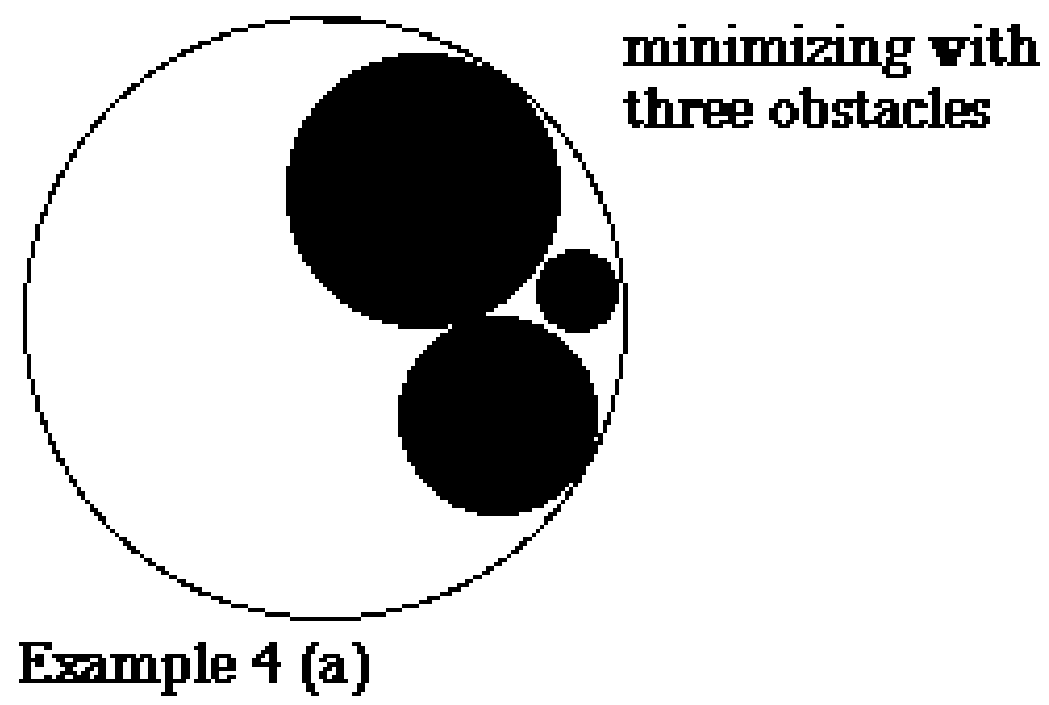

(b) As a variant of (a), we replace the large sphere $\Omega$ with $\Omega^{\prime}:=\Omega \backslash \Upsilon$ where $\Upsilon \subset \Omega_{1}$ for some fixed possible $\Omega_{1}$ as in (a), and is otherwise an arbitrary closed set of positive capacity. We consider $\Omega^{\prime}$ as the exterior region, and insert two spherical obstacles. Then the minimizing configuration is as in Example 3, oriented so that $\Upsilon$ lies within the $\Omega_{1}$ created by the disconnection of $\Omega$. Again, this can be unique or nonunique.

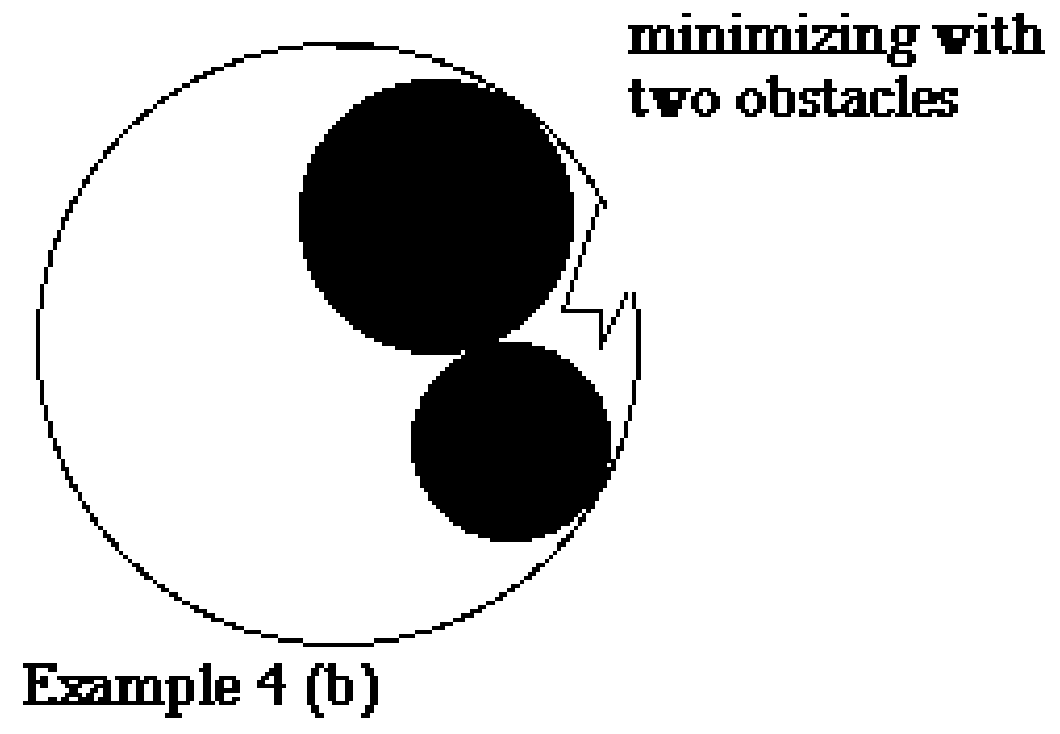

Here we recall the principle of domain monotonicity, which shows that the minimal fundamental eigenvalue for Example 3 is the same as the that of the larger of the two domains into which $\Omega$ is disconnected. In case (a), if the third obstacle were inserted into this domain, the eigenvalue would strictly increase [cf. Co95, McG96, McG98]. On the other hand, the eigenvalue is unaffected in comparison to Example 3, if the third obstacle is inserted into $\Omega_{1}$. Case (b) follows by virtually the same argument, recalling Theorem II.5. 


\section{Example 5.}

Balls with sectors or lines (resp. hyperplane) segments removed.

(a) Consider a domain $\Omega$ formed by removing from a ball of radius $R$ centered at the origin, some subset $\Upsilon$ of a closed sector $S$ symmetric about the $x_{1}$-axis, within the halfplane $x_{1} \geq a \geq 0$; otherwise, $\Upsilon$ is assumed closed and of positive capacity. We call the angle between the edge of the sector and the positive $x_{1}$ axis $\beta$. The obstacle is a hard or soft ball of radius $\rho<\frac{a+R}{2}$.

The maximizing position is then within the triangle (2 dimensions) or cone (3 or more dimensions) bounded by $x_{1}=\frac{a-R}{2}, x_{1}=0$, and the cone with vertex at the origin and making an angle of arccot $\left(\cot \beta+\frac{\mathrm{a}}{\mathrm{R}-\mathrm{a}} \csc \beta\right)$ with the negative $x_{1}$-axis. (Further restrictions on the maximizing position could be precisely formulated if $\rho$ is not sufficiently small.)

The minimizing position(s) are in contact with $S$ (possibly penetrating into its interior).

(b) As a special case of (a), suppose that $\Upsilon$ is a symmetric sector with vertex at $x_{1}=a$, including the limiting case of a line segment. Then we can more precisely say that the maximizing position lies on the negative $x_{1}$ axis with $\frac{a-R}{2}<x_{1}<0$, and the minimizing positions are in contact both with the spherical part of $\partial \Omega$ and with $\partial S$. The minimizing position is not unique.

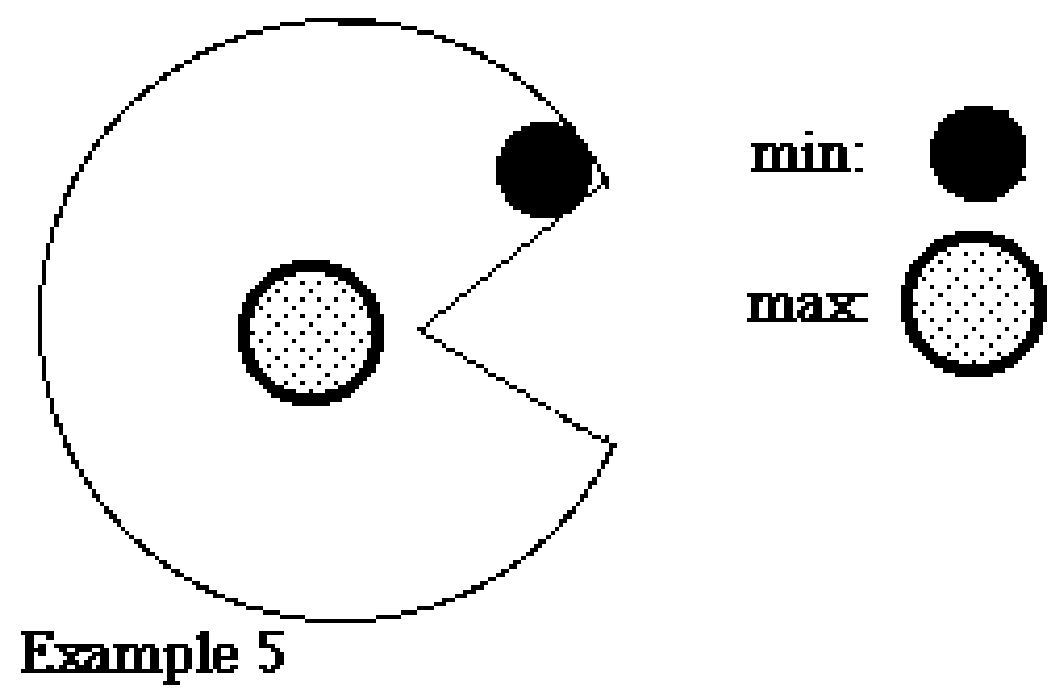

Here we identify as hyperplanes of interior relection: all planes perpendicular to the $x_{1}$-axis with intersection at $-R<x_{1}<\frac{a-R}{2}$; all planes perpendicular to the $x_{1}$-axis with intersection at $0 \leq x_{1}<R$; and all planes through the origin which do not intersect $S$. It is a trigonometric exercise with the latter which leads to the angle identified.

As for case (b), there are additional hyperplanes of interior reflection consisting of all hyperplanes through the origin except those containing the $x_{1}$-axis. 


\section{Example 6.}

Let $\Omega$ be an equilateral $n$-sided polygon, modified by the replacement of its edges with outward circular arcs of equal angular measure $\leq \frac{2 \pi}{n}$, containing a hard or soft circular obstacle $B$. Then the maximizing position $\mathbf{x}$ of the center of $B$ is at the center of the polygon, and the minimizing positions of $B$ put it as near as possible to a vertex.

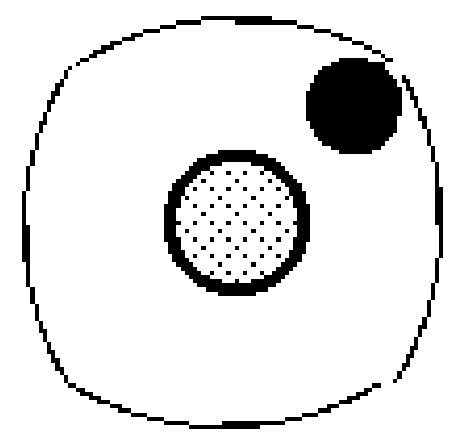

\section{$\min :$}

max

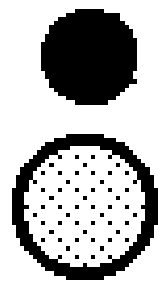

\section{Example 6}

(Straightforward exercise.)

\section{Example 7.}

Let $\Omega$ be a rectangle and suppose that the convex obstacle $B$ has two axes of symmetry parallel to the sides of the rectangle. We consider translati ons of the obstacle $B$. The maximizing position is at the center and the minimizing position is as near as possible to a vertex.

The proof is similar to the proof of Theorem III.4.

\section{Example 8.}

Let $\Omega$ be an equilateral triangle, modified by the replacement of its edges with inward circular arcs of equal angular measure $\leq \frac{\pi}{2}$, containing a hard or soft circular obstacle $B$. Then the maximizing position $\mathbf{x}$ of the center of $B$ is at the center of the triangle, and the minimizing positions of $B$ put it as near as possible to a vertex. 


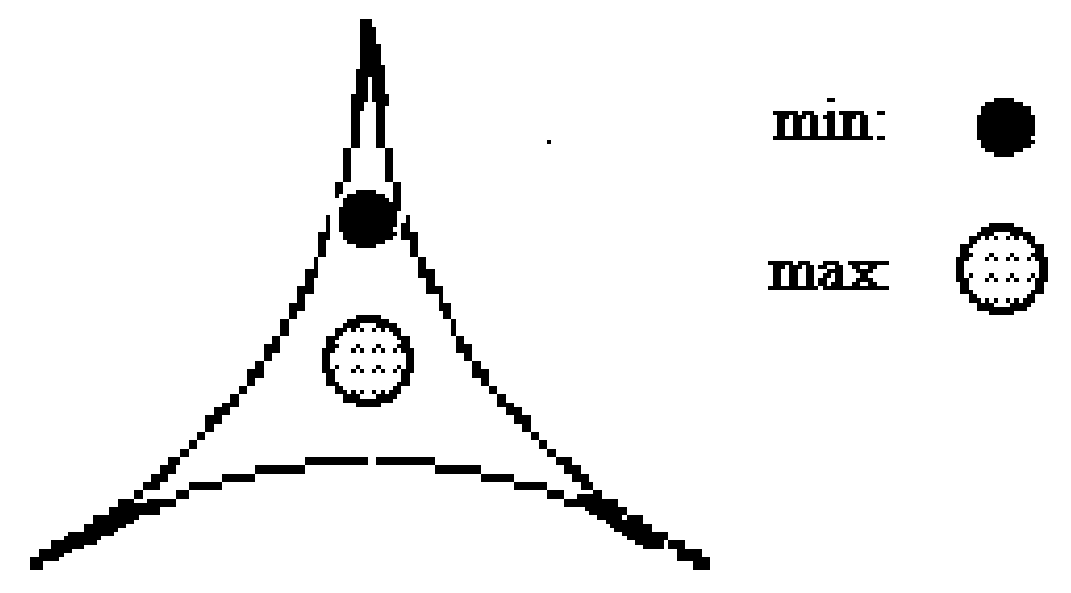

\section{Example 8}

(Straightforward exercise.)

\section{Example 9.}

Let $\Omega$ be the concentric annulus (or spherical shell) of Example 1(e), and suppose that it contains two independently placeable hard obstacles as in Example 3(a). In the maximizing configuration, the two obstacles lie on a common hyperplane which bisects both spheres, and on opposite sides of the center. In the minimizing configuration, either the two obstacles lie on a common hyperplane which bisects both spheres, and on the same side of the center, or else they are in contact with each other (or both).

In this case, unless the obstacles are positioned as claimed, then if one of them is treated as fixed, the hyperplane passing through the origin and through the center of the second obstacle is a hyperplane of interior reflection.

Example 10.

Let $H$ be the portion of a regular helix in $R^{n}, n \geq 3$, at distance $R$ from the $x_{1}$-axis for $-L \leq x_{1} \leq L$. Define $\Omega:=\{\mathbf{x}: \operatorname{dist}(\mathbf{x}, H)<r\}$ for some $r<R$. (This looks like a spring with hemispherical caps on both ends.) Let the hard or soft obstacle be a ball $B$ of radius $\rho<r$. Then the minimizing positions of $B$ put it into contact with either of the two tips where $H$ intersects $\partial \Omega$. At the maximizing position the center $\mathbf{x}$ of $B$ lies on the plane perpendicular to $H$ intersecting $H$ at $x_{1}=0$.

Remark. Moreover, there exists $\rho_{0}>0$, depending on the specific geometry of the helix, such that for $\rho<\rho_{0}$ there is an upper bound on $|\mathbf{x}|$ strictly smaller than the one needed for $B$ to fit within $\Omega$. We do make it precise here.

The proof in this case requires a small twist in the interior-reflection property on which Theorem II.1 relies: Whereas $\Omega$ does not have the standard interior reflection property as given above Theorem II.1, it has the following alternative property: Any hyperplane perpendicular to $H$ other than the one intersecting it at $x_{1}=0$ divides $\Omega$ into two pieces, 
one of which is congruent to a subset of the other, by a half rotation instead of a reflection. (Equivalently, by two reflections.) Applying this operation, rather than a reflection as in the proof of Theorem II.1, we still see that the values of $u$ and $|\nabla u|$ on one half of $\partial B$ dominate those on the other half pointwise, allowing us to show that $\lambda$ is strictly monotonic with respect to displacements tangential to $H$ by (1.2) or, respectively, (1.3).

Our next example involves a soft obstacle, and shows that our analysis is stable with respect to some perturbations of $\Omega$ which destroy both convexity and symmetry.

\section{Example 11.}

$A$ half ellipse with a small handle: Let $\Omega_{0}:=\left\{(x, y) \in R^{2} ; x^{2}+(y / l)^{2}<1, x>0\right\}$ where $l$ is a fixed constant, $l>1$. Now consider the domain $\Omega_{\epsilon}:=$ interior $\left(\Omega_{0} \cup H_{\epsilon}\right)$, where $H_{\epsilon}$ is a "handle" with the following properties:

(i) $H_{\epsilon}$ is a closed subset of $\{x \leq 0\}$

(ii) $H_{\epsilon} \subset\{|y| \leq \epsilon\}$

(iii) $0<\operatorname{vol}(H) \leq C \epsilon$ for some fixed positive constant $C$.

Fix the radius $r<1$ of a spherical soft obstacle $B$, and denote $L_{\Omega_{\epsilon}}:=-\Delta+\alpha \chi_{B}$ on $\Omega_{\epsilon}$. We claim that for all $\epsilon>0$ and sufficiently small, the minimizing obstacle is situated within $\Omega_{0}$ at the greatest possible distance from the $x$-axis. (There are two possibilities, one with $y>0$ and one with $y<0$.)

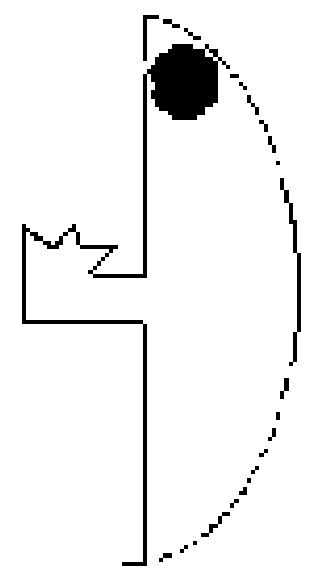

\section{min:}

\section{Example 11}

Proof: We assume that $\epsilon$ is sufficiently small that the obstacle does not fit $\mathrm{i}$ nto the handle. All horizontal lines with $\{|y|>\epsilon\}$ are lines of interior symmetry for $\Omega_{\epsilon}$, so at the minimizing positions for the obstacle, either it is in contact with the boundary or else its center lies within $\{|y| \leq \epsilon\}$. The argument used to prove Theorem III.2 is easily adapted for $\Omega_{\epsilon}$, and shows that the only possible minimizing positions are either as stated in the theorem, i.e., with the maximal value of $|y|$; or else confined to a strip of the form 
$\left\{|y| \leq c_{1} \epsilon\right\}$, where $c_{1}$ is a constant depending only on $l$.

We next eliminate the possible positions in the epsilonic strip by a continuity argument, for we know by the modification of Theorem III.2 that for $\Omega_{0}$, an obstacle in the strip gives rise to a fundamental eigenvalue strictly larger than when the obstacle is at the maximal value of $|y|$ (i.e., the unique two minimizing positions for $\Omega_{0}$ ).

Consider the operator $L_{\Omega}:=-\Delta+\alpha \chi_{B}$ on some domain $\Omega$ with Dirichlet B.C. on $\partial \Omega$ for a ball $B \subset \Omega$ of radius $\mathrm{r}$, and denote respectively by $\lambda_{\Omega}(B)$ and $u_{\Omega, B}(B)(\mathbf{x})$ the first eigenvalue and $L^{2}$-normalized first eigenfunction.

Case 1. Candidate positions where $B$ does not lie wholly inside $\Omega_{0}$. In this case, according to Lemma I.2, the obstacle can be shifted into the interior of $\Omega_{0}$, raising $\lambda$ by a small error. By a variant of the argument for Theorem II.5 and uniform control on $\|u\|_{\infty}$ [e.g., GiTr83, Theorem 8.15], this error tends to 0 as $\epsilon \rightarrow 0$ uniformly in these possible candidate positions. Hence it suffices to consider only candidate positions inside $\Omega_{0}$.

Case 2. Candidate positions inside $\Omega_{0}$ and inside the epsilonic strip $\left\{|y| \leq c_{1} \epsilon\right\}$. Here estimates as in [HiMa91, GeZh94, McG96, McG98] show that $\lambda_{\Omega_{\epsilon}}(B) \rightarrow \lambda_{\Omega_{0}}(B)$ as $\epsilon \rightarrow 0$.

\section{Example 12.}

Example 1(e) revisited: $\Omega$ is a concentric annulus (spherical shell) of outer radius $R$ and inner radius $r$. In it we place a soft spherical obstacle centered at $\mathbf{w}$ and of radius $\rho$; as in 1(e), necessarily, $\rho<\frac{R-r}{2}$. If $r$ is sufficiently small, then at the minimizing position the support $|\mathbf{w}|=R-\rho$, i.e., the obstacle touches the outer boundary of the annulus.

The strategy of the proof is rather general and can be applied to some other cases as indicated below. For this reason we discuss it in some detail. For simplicity, we discuss the case of two dimensions.

Fix the radius $\rho$ of the obstacle and the coupling $\alpha$. We already know that $\mathbf{w} \in \mathcal{C}_{1} \cup \mathcal{C}_{2}$, where

$$
\mathcal{C}_{1}:=\{r+\rho \leq|\mathbf{x}| \leq(R+r) / 2\}, \quad \mathcal{C}_{2}=\{|\mathbf{x}|=R-\rho\}
$$

Note that $R / 2<R-\rho$, and

$$
\mathcal{C}_{1} \subset \mathcal{C}_{1}^{*} \equiv\{\rho \leq|\mathbf{x}| \leq R / 2\}
$$

We denote by $\Lambda$ and $\Lambda^{r}$ the minima of the fundamental eigenvalues given a soft obstacle of radius $\rho$ in domains $\Omega_{0}=\{|x|<R\}$ and $\Omega_{r}$, respectively.

Now we claim the following:

Claim (A): We have $\left|\Lambda-\Lambda^{r}\right| \leq C /|\log r|$, where $C$ is a constant independent of $r$.

Indeed, denoting by $B_{*}^{r}=B_{*, \rho}^{r}$ be the optimal ball for the problem on $\Omega_{r}$, we have $\left.\Lambda^{\epsilon}=\lambda_{\Omega_{r}}\left(B_{*}^{r}\right) \geq \lambda_{\Omega_{0}}\left(B_{*}^{r}\right)\right) \geq \Lambda$ because of domain monotonicity. Here $\lambda_{\Omega}(B)$ is the first 
eigenvalue of $-\Delta+\alpha \chi_{B}$ on $\Omega$. On the other hand, denoting by $B_{*}=B_{*, \rho}$ the optimal ball for the problem on $\Omega_{0}$, we know by [Sw63, Theorem 2], for example, that

$$
\lambda_{\Omega_{r}}\left(B_{*}\right) \leq \lambda_{\Omega_{r}}\left(B_{*}\right)+\frac{C}{|\log r|} .
$$

This yields

$$
\Lambda^{r} \leq \Lambda+\frac{C}{|\log r|}
$$

which implies claim (A).

We know that for $B_{*}^{r}(\mathbf{w}(r))$,

$$
\rho+r \leq|\mathbf{w}(r)| \leq(R+r) / 2, \quad \text { or } \quad|\mathbf{w}(r)|=R-\rho .
$$

If the conclusion did not hold, then there would exist a subsequence $\left\{r_{j}\right\}, r_{j} \rightarrow 0$ and $B^{r_{j}}\left(\mathbf{w}\left(r_{j}\right)\right)$, such that

$$
\rho+r_{j} \leq\left|\mathbf{w}\left(r_{j}\right)\right| \leq\left(R+r_{j}\right) / 2 .
$$

By passing if necessary to a further subsequence, we may assume that $\mathbf{w}\left(r_{j}\right)$ converges to $\mathbf{w}_{0}$ with $\left|\mathbf{w}_{0}\right| \in[\rho, R / 2]$. We use the notation:

$$
B_{0}=B_{\rho}\left(w_{0}\right), \quad B_{*}^{j}=B_{*}^{r_{j}}\left(w\left(r_{j}\right)\right),
$$

for simplicity. Next we claim that

Claim (B): As $j \rightarrow \infty, \lambda_{\Omega_{r_{j}}}\left(B_{*}^{j}\right)-\lambda_{\Omega_{r_{j}}}\left(B_{0}\right) \rightarrow 0$.

Again using $[\mathrm{Sw}]$, we obtain

$$
\left|\lambda_{\Omega_{r_{j}}}\left(B_{0}\right)-\lambda_{\Omega_{0}}\left(B_{0}\right)\right| \rightarrow 0
$$

Granting (B), we get

$$
\left|\lambda_{\Omega_{r_{j}}}\left(B_{*}^{j}\right)-\lambda_{\Omega_{0}}\left(B_{0}\right)\right| \leq\left|\lambda_{\Omega_{r_{j}}}\left(B_{*}^{j}\right)-\lambda_{\Omega_{r_{j}}}\left(B_{0}\right)\right|+\left|\lambda_{\Omega_{r_{j}}}\left(B_{0}\right)-\lambda_{\Omega_{0}}\left(B_{0}\right)\right| \rightarrow 0 .
$$

Combining this with (A), we conclude that

$$
\lambda_{\Omega_{0}}\left(B_{0}\right)=\Lambda
$$

which contradicts the definition of $B_{0}$ and the stated fact about $\Lambda$. Thus, we conclude the desired statement.

It remains only to prove Claim (B):

We shall show that

$$
\left|\lambda_{\Omega_{r_{j}}}\left(B_{*}^{j}\right)-\lambda_{\Omega_{r_{j}}}\left(B_{0}\right)\right| \leq \alpha M\left|B_{*}^{j} \ominus B_{0}\right|^{1 / 2},
$$


where $A \ominus B=(A \backslash B) \cup(B \backslash A)$. As a consequence we obtain: $\lambda_{\Omega_{r_{j}}}\left(B_{*}^{j}\right)-\lambda_{\Omega_{r_{j}}}\left(B_{0}\right) \rightarrow 0$.

First, by definition, $\lambda_{\Omega_{r_{j}}}\left(B_{*}^{j}\right) \leq \lambda_{\Omega_{r_{j}}}\left(B_{0}\right)$. Denote by $\phi_{r_{j}}$ the normalized eigenfunction associated with $\lambda_{\Omega_{r_{j}}}\left(B_{*}^{j}\right)$. Then

$$
\begin{aligned}
\lambda_{\Omega_{r_{j}}}\left(B_{0}\right) & \leq \int_{\Omega_{r_{j}}}\left|\nabla \phi_{r_{j}}\right|^{2}+\alpha \chi_{B_{0}} \phi_{r_{j}}^{2} \\
& =\int\left|\nabla \phi_{r_{j}}\right|^{2}+\alpha \chi_{\left(B_{*}^{j}\right)} \phi_{r_{j}}^{2} d x+\alpha\left(\int_{B_{0}} \phi_{r_{j}}^{2} d x-\int_{B_{*}^{j}} \phi_{r_{j}}^{2} d x\right) \\
& \leq \lambda_{\Omega_{r_{j}}}\left(B_{*}^{j}\right)+\alpha \int_{B_{0} \ominus\left(B_{*}^{j}\right)} \phi_{r_{j}}^{2} d x \\
& \leq \lambda_{\Omega_{r_{j}}}\left(B_{*}^{j}\right)+\alpha\left\|\phi_{r_{j}}\right\|_{L^{4}\left(\Omega_{r_{j}}\right)}^{2}\left|B_{0} \ominus\left(B_{*}^{j}\right)\right|^{1 / 2} .
\end{aligned}
$$

It is easy to see that $\lambda_{\Omega_{r_{j}}}\left(B_{*}^{j}\right)$ is bounded, and hence that $\int_{\Omega_{r_{j}}}\left|\nabla \phi_{r_{j}}\right|^{2} \leq M$. By the Sobolev embedding theorem, we obtain $\left\|\phi_{r_{j}}\right\|_{L^{4}\left(\Omega_{r_{j}}\right)}^{2} \leq M$, yielding the desired estimate.

The strategy of the perturbation argument used for Example 12 can be used in many other situations. To summarize: Let $\Omega_{\epsilon}$ be a (singular) perturbation of $\Omega$ and assume, for simplicity, $\Omega_{\epsilon} \subset \Omega$. For a fixed $D \subset \Omega$, assume that $\lambda^{\epsilon}(D) \rightarrow \lambda(D)$ as $\epsilon \rightarrow 0$. Here $\lambda^{\epsilon}(D)$ and $\lambda(D)$ are the fundamental eigenvalues of $-\Delta+\alpha \chi_{D}$ on $\Omega_{\epsilon}$ and $\Omega$, respectively. We denote by $\Lambda=\lambda\left(B\left(x_{0}\right)\right)$ and $\Lambda_{\epsilon}=\lambda\left(B\left(x_{\epsilon}\right)\right)$ the optimal eigenvalues on $\Omega_{\epsilon}$ and $\Omega$, respectively. Then, if $x_{0} \in \mathcal{C}_{0}$ and $x_{\epsilon} \in \mathcal{C}_{0} \cup \mathcal{C}_{\epsilon}$ with $\mathcal{C}_{\epsilon} \subset \mathcal{C}$, and if $\inf _{y \in \mathcal{C}} \lambda(B(y))>\Lambda$, then $x_{\epsilon} \in \mathcal{C}_{0}$ for sufficiently small $\epsilon$.

We close by stating another perturbative result related to Examples 5 and 12:

\section{Example 13.}

In two dimensions, let $\Omega_{\epsilon}=\{\epsilon<|x|<b\} \backslash\{(r, \theta) ; \epsilon<r<b,|\theta| \leq \beta\}$ with $\beta<\pi / 2$, and suppose that it contains a soft spherical obstacle. Then for sufficiently small $\epsilon$, at the minimizing position the obstacle is as close as the corner points $(r, \theta)=(b, \pm \beta)$.

\section{Acknowledgments}

This collaboration was made possible by two visiting invitations, that of Pawel Kröger to the Georgia Institute of Technology in Atlanta in April, 1995, and that of Evans Harrell and Kazuhiro Kurata to the Erwin Schrödinger Institut in Vienna in May, 1998. We are grateful to those institutions, as well as the French C.N.R.S. and the University of Toulouse 1 and 3, for hospitality. Moreover, we wish to thank Ivor McGillivray for useful references.

\section{Bibliography}

[Al60] A.D. Alexandrov, Certain estimates for the Dirichlet problem, Soviet Math Doklady 1 (1960).

[As99] M.S. Ashbaugh, private commmunication. 
[BeNi91] H. Berestycki and L. Nirenberg, On the method of moving planes and the sliding method, Bol. Soc. Bras. Mat. 22 (1991) 1-37.

[CGIKO99] S. Chanillo, D. Grieser, M. Imai, K. Kurata, and I. Ohnishi, Symmetry breaking and other phenomena in the optimization of eigenvalues for composite membranes, Commun. Math. Phys., to appear.

[Co94] A. Colesanti, A variational problem for harmonic functions in ring-shaped domains with partially free boundary, SIAM J. Math. Anal. 25 (1994) 1122-1127.

[Co95] G. Courtois, Spectrum of manifolds with holes, J. Funct. Anal. 134 (1995) 194-221.

[Da95] E.B. Davies, Spectral Theory and Differential Operators, Cambridge Studies in Advanced in Mathematics 42. Cambridge: Cambridge University Press, 1995.

[F193] M. Flucher, An asymptotic formula for the minimal capacity among sets of equal area, Calculus of Variations 1 (1993), 71-86.

[F195] M. Flucher, Approximation of Dirichlet eigenvalues on domains with small holes, J. Math. Anal. Appl. 193 (1995) 169-199.

[GaSc53] P.R. Garabedian and M. Schiffer, Convexity of domain functionals, J. Anal. Math. 2 (1952-53) 281-368. (receipt date is 1953).

[GeZh94] F. Gesztesy and Z. Zhao, Domain perturbations, Brownian motion, capacities, and ground states of Dirichlet Schrödinger operators, Math. Z. 215 (1994) $143-150$.

[GiTr83] D. Gilbarg and N.S. Trudinger, Elliptic Partial Differential Equations of Second Order, second edition, Grundlehren der mathematischen Wissenschaften 224. Berlin, Heidelberg, New York, London, Paris, Tokyo: Springer-Verlag, 1983

[Ha08] J. Hadamard, Mémoire sur le problème d'analyse relatif à l'équilibre des plaques élastiques encastrées, Méms. prés. par div. savants a l'Acad. des Sci. 33 (1908) $1-128$.

[HiMa91] P.D.Hislop and A. Martinez, Scattering Resonaces of a Helmholtz Resonator, Indiana Univ. Math. J. 401991 767-788.

[JeKe85] D.Jerison and C.Kenig, Unique continuation and absence of positive eigenvalues for Schrödinger operators, Ann. Math. 121(1985) 463-494.

[McG96] I. McGillivray, Capacitary estimates for Dirichlet eigenvalues, J. Funct. Anal. 139 (1996) 244-259.

[McG98] I. McGillivray, Capacitary asymptotic expansion of the groundstate to second order, Commun. PDE. 23, 11/12 (1998) 2219-2252

[PrWe84] M.H. Protter and H.F. Weinberger, Maximum Principles in Differential Equations, New York: Springer-Verlag, 1984. 
[ReSi78] M. Reed and B. Simon, Methods of Modern Mathematical Physics, IV. Analysis of Operators, New York: Academic Press, 1978.

[Se71] J. Serrin, A symmetry problem in potential theory, Arch. Rat. Mech. Anal. 43 (1971) 304-318.

[Sw63] C.A. Swanson, Asymptotic variational formulae for eigenvalues, Canad. Math. Bull. 6 (1963) 15-25. 